\title{
A quantitative approach towards a better understanding of the dynamics of Salmonella spp. in a pork slaughter-line.
}

H. A. M. van Hoek, Angela; Jonge, Rob de; M. van Overbeek, Wendy; Bouw, El; Pielaat, Annemarie; Smid, Joost H.; Malorny, Burkhard; Junker, Ernst; Löfström, Charlotta; Pedersen, Karl

Total number of authors:

12

Published in:

International Journal of Food Microbiology

Link to article, DOI:

10.1016/j.ijfoodmicro.2011.10.013

Publication date:

2012

Link back to DTU Orbit

Citation (APA):

H. A. M. van Hoek, A., Jonge, R. D., M. van Overbeek, W., Bouw, E., Pielaat, A., Smid, J. H., Malorny, B., Junker, E., Löfström, C., Pedersen, K., Aarts, H. J. M., \& Heres, L. (2012). A quantitative approach towards a better understanding of the dynamics of Salmonella spp. in a pork slaughter-line. International Journal of Food Microbiology, 153(1-2), 45-52. https://doi.org/10.1016/j.ijfoodmicro.2011.10.013

\section{General rights}

Copyright and moral rights for the publications made accessible in the public portal are retained by the authors and/or other copyright owners and it is a condition of accessing publications that users recognise and abide by the legal requirements associated with these rights.

- Users may download and print one copy of any publication from the public portal for the purpose of private study or research.

- You may not further distribute the material or use it for any profit-making activity or commercial gain

- You may freely distribute the URL identifying the publication in the public portal 
1 A quantitative approach towards a better understanding of the dynamics of

2 Salmonella spp. in a pork slaughter-line.

3 Angela H. A. M. van Hoek ${ }^{a, b}, *$, Rob de Jonge ${ }^{b}$, Wendy M. van Overbeek ${ }^{a, b}$, El Bouw ${ }^{\text {a,b }}$, 4 Annemarie Pielaat ${ }^{\mathrm{b}}$, Joost H. Smid ${ }^{\mathrm{b}}$, Burkhard Malorny ${ }^{\mathrm{c}}$, Ernst Junker ${ }^{\mathrm{c}}$, Charlotta Löfström 5 d, Karl Pedersen ${ }^{d}$, Henk J. M. Aarts ${ }^{\text {a,b }}$, Lourens Heres ${ }^{\text {e }}$

6

a RIKILT - Institute of Food Safety, Wageningen UR, Akkermaalsbos 2, 6708 WB Wageningen, The Netherlands

${ }^{b}$ National Institute for Public Health and the Environment (RIVM), Centre for Infectious Disease Control (CIb), Antonie van Leeuwenhoeklaan 9, 3721 MA Bilthoven, The Netherlands

* Corresponding author: National Institute for Public Health and the Environment, Centre for Infectious Disease Control, Laboratory for Zoonoses and Environmental Microbiology. P.O. Box 1, 3720 BA Bilthoven, The Netherlands. Phone: +31-30-2747058, Fax: +31-30-2744434, E-mail: angela.van.hoek@rivm.nl. 


\section{ABSTRACT}

Pork contributes significantly to the public health disease burden caused by Salmonella infections. During the slaughter process pig carcasses can become contaminated with Salmonella. Contamination at the slaughter-line is initiated by pigs carrying Salmonella on their skin or in their faeces. Another contamination route could be resident flora present on the slaughter equipment. To unravel the contribution of these two potential sources of Salmonella a quantitative study was conducted. Process equipment (belly openers and carcass splitters), faeces and carcasses (skin and cutting surfaces) along the slaughter-line were sampled at eleven sampling days spanning a period of 4 months.

Most samples taken directly after killing were positive for Salmonella. On $96.6 \%$ of the skin samples Salmonella was identified, whereas a lower number of animals tested positive in their rectum (62.5\%). The prevalence of Salmonella clearly declined on the carcasses at the re-work station, either on the cut section or on the skin of the carcass or both $(35.9 \%)$. Throughout the sampling period of the slaughter-line the total number of Salmonella per animal was almost $2 \log$ lower at the re-work station in comparison to directly after slaughter.

Seven different serovars were identified during the study with $S$. Derby (41\%) and $S$. Typhimurium (29\%) as the most prominent types. A recurring $S$. Rissen contamination of one of the carcass splitters indicated the presence of an endemic 'house flora' in slaughterhouse studied. On many instances several serotypes per individual sample were found.

The enumeration of Salmonella and the genotyping data gave unique insight in the dynamics of transmission of this pathogen in a slaughter-line. The data of the presented study support the hypothesis that resident flora on slaughter equipment was a relevant source for contamination of pork.

Keywords: Salmonella; pigs; PCR; quantitative; resident flora; slaughterhouse. 


\section{INTRODUCTION}

Salmonellosis is an important cause of food-borne human gastroenteritis in most European countries (EFSA, 2010; Valkenburgh et al., 2007). Farm animals and foods of animal origin form an important source of human Salmonella infections. In various European countries a significant number of human cases of salmonellosis (up to $25 \%$ ) is described to be related to the consumption of pork and pork products (EFSA, 2006; van Pelt et al., 2000; Valdezate et al., 2005).

Carrier pigs are a predominant source of Salmonella contamination of pig carcasses during the slaughtering process (Alban and Stärk, 2005; Baptista et al., 2010; Berends et al., 1997; Borch et al., 1996;). Pigs may already have Salmonella on their skin before entering a slaughterhouse and, despite stringent hygiene procedures during carcass processing, cross contamination to both Salmonella positive and - negative carcasses can occur. The slaughterline itself can become contaminated by faeces of carrier pigs. In addition, the presence of endemic 'house flora' of Salmonella has been described for several slaughterhouses (Baptista et al., 2010; Hald et al., 2003; Visscher et al., 2011; Warriner et al., 2002).

European data on the prevalence of Salmonella contaminated carcasses and on serotypes of Salmonella on the carcasses is available in various papers. For example, Hald et al. (2003) documented that the prevalence of Salmonella contaminated carcasses varied between 0 and 8.5\% among 1,623 carcasses examined from five different countries. An EFSA study (26 countries; 5,736 carcass samples) reported a prevalence of Salmonella positive carcasses of 0-20\% (EFSA, 2008). The most frequently isolated serotype in both studies was $S$. Typhimurium.

The aim of this study was to investigate the dynamics of Salmonella in a pig slaughtering process and to assess the origin of carcass contamination. Hereto, the prevalence of Salmonella contaminated carcasses was determined. In addition, the concentration of this 
pathogen was measured at different sites on the pork meat and slaughtering equipment throughout the slaughtering-line by sampling individual carcasses at exsanguination up to the re-work station. Salmonella isolates were serotyped and genotyped. 
MATERIALS AND METHODS

\section{Slaughterhouse characteristics}

The Dutch slaughterhouse investigated in this study was partly automated with robots for pre-cutting, belly opening, rectum drilling, splitting, leaf lard removal, neck cutting and marking. The capacity of the slaughterhouse is 650 pigs per hour, and 5,000-6,000 animals per day. The waiting time for the pigs at the slaughterhouse was as short as possible (not more than $2 \mathrm{~h}$ ). Before entering the slaughter-line pigs were electrically stunned, sticked on a table, scalded in a tank, dehaired, flamed, wet polished, flamed and wet polished for a second time.

The belly opener cuts open the belly of a carcass and then cleaves the breastbone into two symmetrical parts. The carcass splitter cuts a carcass into two equal halves with a double knife, without cutting the head.

\section{Sampling strategy}

Carcass and equipment samples were collected on eleven days over a period of four months. Different herds were sampled on one sampling day, with a preference of two animals per herd, to account for herd variability. In total, 118 pigs and their carcasses were sampled at two steps of the slaughter process (see Fig. 1 for exact sampling sites)). Directly after exsanguination, skin and rectal samples were taken for the detection, enumeration and typing of Salmonella. Immediately after exsanguination 4 cork borer samples were obtained from the shoulder of the animal. A sterile hand held cork borer was used to make four incisions on the shoulder. With a sterile scalpel and forceps slices of $5 \mathrm{~cm}^{2}$ with a thickness of approximately $5 \mathrm{~mm}$, were cut from the carcass. The four tissue samples, representing a total of $20 \mathrm{~cm}^{2}$, were collected in one sterile plastic bag, constituting one sample. In addition, a rectal sample was taken from the same animal with a sterile swab (Transwab, Medical Wire and equipment Co. 
Ltd., Corsham, Wilts., England), which was immediately placed in $6 \mathrm{ml}$ Buffered Peptone Water (BPW; bioTRADING Benelux B.V., Mijdrecht, The Netherlands).

The carcasses sampled at exsanguination were tracked in the slaughter-line and sampled again after meat inspection at the re-work station. From the cutting site, ham, back before pelvis, sternum and shoulder muscle were sampled with the cork borer. From the lard side, samples were taken with the cork borer from the back, the jowl, the ham and the belly. These interior and exterior samples were collected separately in two sterile plastic bags. In this way a paired set of $2 \times 2$ different samples were obtained from each animal; two at exsanguination (shoulder (EE), faeces (FS)) and two after final meat inspection at the re-work station (exterior (RE), interior (RI)).

In the slaughter-line the sets of parallel operating belly openers (BO) as well as the carcass splitters (CS) were sampled prior to the start and at the end of the day, immediately after finishing with the slaughtering process. Blades and other easy to reach contact surfaces from the belly openers and the splitting robots were swabbed on both sites using the Meat/Turkey carcass sampling kit (Nasco, Fort Atkinson, WI). In addition, sterile flexistem brushes were used for sampling of parts of the equipment which were less accessible with the carcass sampling kit.

All samples were cooled on site and transported to the laboratory to be analysed on the same day of collection.

\section{Detection of Salmonella}

Cork borer samples were weighed after arrival in the laboratory and an equal volume of BPW was added. To rectal swabs, equipment swabs and flexistem brushes 6,20 , and $40 \mathrm{ml}$ of BPW, respectively, was added. Cork borer and equipment swab samples were homogenised for 1 min with a Stomacher 400 (Seward, Worthing, United Kingdom). Rectal swabs and 
equipment samples taken with a flexistem brush were vortexed for $30 \mathrm{~s}$. A $5 \mathrm{ml}$ aliquot was removed from each sample and stored at $4{ }^{\circ} \mathrm{C}$ for enumeration later (see next section). After addition of $90 \mathrm{ml} \mathrm{BPW}$ to the cork bore samples, rectal and equipment swabs, all samples were incubated without shaking at $37^{\circ} \mathrm{C}$ for 18 to $20 \mathrm{~h}$.

DNA was isolated from a $1 \mathrm{ml}$ aliquot of the enriched culture, using a Chelex-100 suspension (50-100 mesh; Bio-Rad Laboratories B.V., Veenendaal, The Netherlands) according to the manufacturer's instructions. From the final DNA solution, a $5 \mu \mathrm{l}$ aliquot was directly used as template in the PCR assay described below.

The Salmonella real-time assay described by Malorny et al. (2004), except for the internal amplification control, was used to determine the presence of DNA of this pathogen in the various samples. The $50 \mu \mathrm{l}$ PCR mixture contained $0.4 \mu \mathrm{M}$ of the primers ttr-4 and ttr- $6,0.25$ $\mu \mathrm{M}$ ttr-5 probe (5'-FAM, 3'-BHQ1), 1×Universal Mastermix (Diagenode sa, Liège, Belgium) and a $5 \mu 1$ aliquot of the sample DNA. Conditions for the real-time PCR were $95{ }^{\circ} \mathrm{C}$ for $1 \mathrm{~min}$ followed by 45 cycles of $95{ }^{\circ} \mathrm{C}$ for $15 \mathrm{~s}$ and $65^{\circ} \mathrm{C}$ for $30 \mathrm{~s}$. PCR tests were performed on a $\mathrm{iQ}^{\mathrm{TM}} 5$ Cycler (Bio-Rad Laboratories B.V., Veenendaal, The Netherlands) and data was analysed using the Bio-Rad iQ5 software (Version 2.0).

Samples that were found positive by PCR were considered to be true positives for the assessment of the Salmonella prevalence (the cut off value was set at threshold cycle Ct 40 as result of an internal house validation process).

\section{Enumeration of Salmonella}

The most probable number (MPN; de Man, 1983) method was used to estimate Salmonella numbers in the samples identified as positive by PCR. Three subsequent 10 -fold serial dilutions were prepared from the stored $5 \mathrm{ml}$ of the original samples. In triplicate $1 \mathrm{ml}$ of each dilution was added to $9 \mathrm{ml}$ of BPW and enriched for $18 \pm 2 \mathrm{~h}$ at $37^{\circ} \mathrm{C}$. Three separate and 
equally spaced drops of incubated BPW (total $100 \mu \mathrm{l}$ ) were pipetted onto the surface of a Modified Semi-solid Rappaport Vassiliadis (MSRV) medium base plate (Merck B.V., Schiphol-Rijk, The Netherlands) supplemented with Novobiocin (20 mg ${ }^{-1}$ ) (Oxoid B.V., Badhoevedorp, The Netherlands) in a triangular configuration. MSRV plates were incubated at $41.5{ }^{\circ} \mathrm{C}$ and examined after 24 and $48 \mathrm{~h}$ for suspect Salmonella growth. A sterile loop (1 $\mu 1)$ was dipped into the edge of any opaque growth and streaked onto $\mathrm{SM}^{\circledR}$ ID2 agar plates (BioMérieux SA, Marcy l'Etoile, France) which were incubated at $37{ }^{\circ} \mathrm{C}$ for $24 \mathrm{~h}$ for the confirmation of Salmonella.

To compute the MPN per ml of BPW, it was assumed that all Salmonella were detached from the cork borer sample of the carcass surface and brought into the BPW during stomaching. The MPN per $\mathrm{ml}$ were converted to MPN per $\mathrm{cm}^{2}$. Hereto, it was assumed that the bacteria were homogeneously spread over the carcass skins. Salmonella numbers per gram of faeces from the rectal swab data were also assessed. The amount of faeces on the swab was not determined during the sampling experiment. Therefore, a small study was performed afterwards in which 50 swabs were weighted before and after insertion into pigs' rectums. The mean amount of faeces that was found on a swab was used to estimate the number of Salmonella per gram faeces, using the MPN per swab.

\section{Statistical data analysis}

A beta distribution was used to describe uncertainty about the prevalence estimates of Salmonella on site or at day level (Vose, 2000). For further analysis of the quantitative Salmonella data, the hypothesis that the variation in the Log of all MPN data at one sampling site for Salmonella-positive carcasses can be expressed by a Normal distribution was verified by visually checking its fit to Normality in a quantile-quantile plot. If, by this test, no deviations from normality could be seen, then the per day variation in the MPN data from one 
sampling site was expressed by a Log-Normal $(\mu, \sigma)$ distribution. The parameters of this distribution were estimated using maximum likelihood estimation, yielding the estimators $\hat{\mu}$ (mean) and $\hat{\sigma}$ (standard error). Samples that were positive by PCR, but in which no Salmonella was detected in the dilution series for the MPN assessment, were taken into account and regarded as censored positives. For the censored numbers, the cumulative LogNormal $(\mu, \sigma)$ distribution function was used to represent the probability of being an observation below detection limit (Gelman et al., 2004). Such concentration distributions could, however, not be assessed for all days. If most, or all, samples were negative in the MPN dilution series on one day, then $\hat{\mu}$ and $\hat{\sigma}$ could not be estimated. For such data sets only the upper limit of the expected concentration $\hat{\mu}$, as provided by the minimal MPN, is given.

\section{Sero- and genotyping of Salmonella}

Depending on the Salmonella concentrations, one to a maximum of five (representative) isolates from each sample were randomly selected. All isolates were stored at $-70{ }^{\circ} \mathrm{C}$ until use.

The multiplex PCR described by Lim et al. (2003) was used to discriminate between $S$. Typhimurium and non-Typhimurium serotypes in the numerous isolates from the slaughterhouse. The non-Typhimurium isolates were subsequently serotyped by slide and tube agglutination following the Kauffmann-White scheme (Grimont and Weill, 2007).

Multiple-locus variable-number of tandem-repeat analysis (MLVA) was performed on the (monophasic) S. Typhimurium isolates as described previously (Torpdahl et al., 2007) to determine whether the isolates were epidemiologically related. Only one (monophasic) $S$. Typhimurium isolate per sample was analysed by MLVA. The MLVA repeats were calculated and named according to the method described by Lindstedt et al. (2004). 
Pulsed-field gel electrophoresis (PFGE) was carried out on $S$. Derby and $S$. Rissen isolates with the XbaI restriction enzyme according to the Pulse-Net protocol (Ribot et al., 2006). Gels were analysed using BioNumerics 6.5 software. A dendrogram was produced using the limit and $1.5 \%$ optimisation. 


\section{RESULTS}

\section{Salmonella screening and enumeration}

Salmonella was identified on the skin surfaces of $96.6 \%$ of all carcasses sampled at exsanguination (Table 1). The estimated mean concentration $(\hat{\mu})$ of Salmonella per day in the samples at this site varied between 0.04 and $1.75 \log \mathrm{MPN} \mathrm{cm}{ }^{-2}$ (Table 2). Of the rectal swabs taken directly after exsanguination $62.5 \%$ were identified positive, whereas the average number of Salmonella was $1.88 \pm 1.42 \log$ MPN g. ${ }^{-1}$. At the re-work station, $16.2 \%$ and $29.9 \%$ of the exterior and interior samples, respectively, were tested positive for Salmonella (Table 1). In addition, the pathogen counts were lower in comparison to samples taken at exsanguination, with maximum estimated numbers of Salmonella of 0.11 and $-0.13 \log$ MPN $\mathrm{cm}^{-2}$ on the carcass surface (exterior) and cut section (interior), respectively (Table 2). Of all the samples taken in this study, $44.5 \%$ (265/596) were identified as Salmonella positive. The prevalence of Salmonella on the different carcass sampling sites varied between sampling days (Table 1). For the carcass samples collected at the re-work station, an increase in Salmonella prevalence was observed from around the second half of the sampling period (0806-2009 till 16-06-2009), especially for samples collected from the interior part of the carcass. The prevalence declined again towards the end of the experiment. Within one day no clear increase of Salmonella positive samples could be demonstrated, i.e. the prevalence of this pathogen in samples taken in the morning were not different from those obtained in the afternoon (Fig. 2).

Before slaughter, no Salmonella could be demonstrated on either belly openers, whereas at the end of slaughter 3 out of 40 samples (7.5\%) were tested positive. On one sampling day, Salmonella was identified on both belly openers (Table 1).

Samples taken from the carcass splitters were more frequently found to harbour Salmonella. More specifically, carcass splitter number 2 (CS2) was repeatedly contaminated with this 
pathogenic microorganism. In total, during eight out of the eleven sampling days Salmonella was identified on this robot after the end of slaughter. Moreover, on two consecutive days Salmonella was already found on carcass splitter 2 at the beginning of the slaughtering process (Table 1). In all cases, equipment swabs and flexistem brushes had equal test results.

\section{Salmonella serotypes}

In total, 620 Salmonella isolates were obtained from all samples taken during this study. Because $S$. Typhimurium was expected to be the most prevalent serovar in pigs (Hald et al, 2003; EFSA, 2008), the multiplex PCR described by Lim et al. (2003) was used to discriminate $S$. Typhimurium isolates from other serovars. The PCR results revealed that $67.5 \%$ of all salmonellae isolated at the slaughterhouse were non-Typhimurium isolates. Because of this very large set, it was decided to serotype the main part (64\%). When not all isolates from one sample were typed, the result of the subset of typed isolates was assumed to reflect the serotypes of the non-typed ones.

Overall, seven different serotypes were identified, i.e. S. 4,5,12:i:- (from here on called monophasic $S$. Typhimurium), $S$. Bredeney, $S$. Brandenburg, $S$. Derby, $S$. Infantis, $S$. Rissen and $S$. Typhimurium (Table 3). Six serotypes were characterised from the animals entering the slaughterhouse, whereas only five different serovars were identified on the carcasses after slaughtering, and only three serotypes were isolated from the slaughterhouse equipment sampled. The most prominent serovars identified at the carcass at exsanguination and their rectal swabs were $S$. Derby (38\%), S. Typhimurium (36\%) and $S$. Brandenburg (18\%) (Table 4). The serotypes frequently isolated from the carcasses at the end of the slaughter-line were $S$. Derby (47\%) and $S$. Rissen (25\%), whereas $S$. Typhimurium was only found in $18 \%$ of the cases. The predominant Salmonella serotype isolated at the slaughterhouse varied by day of the study. 
Although $S$. Typhimurium was prominently present on the carcasses at exsanguination and to a lesser extent at the re-work station, this serovar was not isolated from the carcass splitters. In contrast on the belly openers $S$. Typhimurium was found in two out of three occasions. Carcass splitter 2 (CS2) was frequently contaminated with serovars Derby (56\%) and Rissen $(44 \%)$.

In $15 \%$ of all Salmonella positive incidences multiple serovars were isolated from individual samples. This was especially true for carcasses at exsanguination (data not shown).

\section{Salmonella genotypes}

At least one $S$. Typhimurium or monophasic $S$. Typhimurium isolate from each individual swab or carcass sample (80 animals, 119 isolates in total) positive for these serovars was typed by multiple-locus variable-number of tandem-repeat analysis (MLVA). Nineteen and three different MLVA types could be distinguished among the $S$. Typhimurium and monophasic $S$. Typhimurium isolates analysed, respectively (Table 5).

In 18 cases the same MLVA type was detected in both the rectal swab and exterior sample at exsanguinations, whereas 5 times different MLVA types were encountered in these samples. The $17 S$. Typhimurium and monophasic $S$. Typhimurium isolates originating from carcasses at the re-work station matched with MLVA types isolated at exsanguination from the same animals, except in three instance (Table 6; Animals 149_1, 657_1 and 657_2).

The two $S$. Typhimurium MLVA types detected on belly opener 2 (BO2) were also found on Salmonella samples originating from the incoming animals on those sampling days. In addition, both of these MLVA types were identified in samples taken at the re-work station (Table 5).

A selection of the $S$. Derby and $S$. Rissen isolates $(\mathrm{n}=96)$ were genotyped using PGFE. The dendrogram (Fig. 3) shows that the $S$. Rissen isolates belonged to one indistinguishable type, 
whereas the PFGE profiles varied among the $S$. Derby isolates analysed, although one particular $S$. Derby genotype clearly dominated the phylogenetic tree. Isolates belonging to this branch originated from various sampling days and all types of samples taken at the slaughterhouse, except the belly opener. In contrast, one branch with a PFGE pattern very similar to the $S$. Rissen profile contained $5 S$. Derby isolates isolated only from the carcass splitter but at different sampling days.

From several individual carcasses, $S$. Derby was isolated at two or more sampling sites (Table 4). The phylogenetic tree in Figure 3 includes some of these isolates (in bold). $S$. Derby isolates originating from rectal swabs (FS) and skin samples (EE) showed an identical PFGE pattern in $75 \%$ of the cases $(n=4)$, whereas, only different PFGE profiles were encountered among the exsanguination (EE) and the re-work station isolates of the same animal of this serovar $(n=8)$. 


\section{DISCUSSION}

The prevalence of Salmonella contaminated carcasses started with $96.6 \%$ at exsanguination and was $35.9 \%$ after slaughtering at the re-work station. The level of contaminated carcasses in this study was relatively high, compared to other studies (Bouvet et al., 2003; de Busser et al., 2011; Swanenburg et al., 2001a). This high level of Salmonella positive samples gave the opportunity to get a clear picture of the contamination routes.

At the re-work station, over $35 \%$ of the carcasses tested were Salmonella positive. In $10.3 \%$ of all tested carcasses, Salmonella was detected on both the cut section and on the skin, 19.7\% of the tested carcasses were only contaminated at the cut section, and $6.0 \%$ contained Salmonella only on the skin. So the slaughter process reduces the number of skin contaminated carcasses from 96.6 to $16.2 \%$. Cross contamination via the slaughter process was responsible for at least $30 \%$ of all carcasses, i.e. the carcasses were contaminated at the interior side. These results correspond to data reported by others (Berends et al., 1997; Botteldoorn et al., 2003). However, this cross contamination percentage might be an underestimated value since they do not take into account the genotypic diversity of Salmonella serovars. In the present study on the one hand the same MLVA type was found at exsanguination and re-work station (Table 6), but on the other hand it was clearly shown that genotypically different subtypes of the same Salmonella serotype can be present on one carcass at exsanguination and at the re-work station (see Fig 3 and Table 5).

An excision technique was used as the sampling method for pig skins and carcasses. In many studies (Botteldoorn et al, 2003; EFSA, 2008; Hald et al., 2003; Oosterom et al., 1985; Swanenburg et al., 2001a, 2001b) dry-wet swabbing was the technique of choice. Comparison of both techniques showed that the excision technique was approximately 10-fold more sensitive, but there seemed to be no linear relationship between the two results (Hutchison et al, 2005; Martínez et al., 2010). In case of low concentrations, swabbing a large area is to be 
preferred above excision of a small area (Lindblad, 2007), since the excision techniques only samples $5 \mathrm{~cm}^{2}$ per excision. The concentration data obtained in this study clearly showed that the level of contamination of the sampled carcasses was high enough to use the excision technique.

The average number of Salmonella per carcass was almost $2 \log$ lower at the end of the slaughter-line. On the skin $\left(12,000 \mathrm{~cm}^{2}\right)$ a 10 fold lower number was found, i.e. 3.8 to 0.37

Salmonella per $\mathrm{cm}^{2}$. At the cutting area $\left(3,000 \mathrm{~cm}^{2}\right)$, the average MPN of Salmonella was 0.48 per $\mathrm{cm}^{2}$. As a consequence, the average number of Salmonella per carcass decreased from 44,050 (prevalence $\times$ concentration $\times$ surface; $0.966 \times 3.8 \times 12,000)$ at exsanguination to 1,150 per carcass $(0.162 \times 0.37 \times 12,000+0.299 \times 0.48 \times 3,000)$ at the re-work station. As $37.5 \%$ of all salmonellae on carcasses at the re-work station were found on the cutting edges, cross contamination is responsible for more than $35 \%$ of all Salmonella on pork carcasses based on bacterial counts.

The seven Salmonella serovars identified in this study, i.e. S. Bredeney, S. Brandenburg, $S$. Derby, S. Infantis, monophasic S. Typhimurium (Salmonella 4,5,12:i:-), S. Rissen and $S$. Typhimurium were also described by various other authors on pigs at the slaughterhouse stage (Arguello et al., 2011; Bouvet et al., 2003; de Busser et al., 2011; Hald et al., 2003; Swanenburg et al., 2001a).

At the re-work station, five different serovars were detected, whereas at exsanguination six Salmonella serotypes were characterised (Table 3 and 4). Two serovars detected at exsanguination, i.e. $S$. Bredeney and $S$. Infantis, were not detected at the re-work station. It might be possible that the contamination level with these serovars was very low and that they disappeared during the slaughter process. In contrast, one serovar, i.e. S. Rissen, was not detected at exsanguination but was detected at the re-work station and on one of the carcass splitters. The companies own monitoring program reflected that this slaughterhouse 
encountered hygiene problems during and after the study (data not shown). The serological pattern (Table 3 and 4) clearly indicated complicated contamination routes.

The phenomenon of multiple serovars present in individual samples (15\%), especially in those taken from carcasses at exsanguination suggested an underestimation of Salmonella serotypes in pork, since routinely only one isolate per sample is serotyped.

In order to determine their origin, isolates of the serovars $S$. Rissen, $S$. Derby and (monophasic) $S$. Typhimurium were subtyped. The results differed per serotype. S. Rissen was not detected on any of the incoming pigs. Only one PFGE genotype was found on cutting areas of carcasses at the re-work station and on the carcass splitter on various sampling days. This result strongly suggested that resident house flora was a source of carcass contamination. $S$. Derby showed the characteristics of a cross contaminator as none of the strains detected on a single carcass at the re-work station was detected on the same carcass at exsanguination. Comparing MLVA types of (monophasic) $S$. Typhimurium isolates on carcasses at exsanguination and re-work station revealed that (monophasic) $S$. Typhimurium can originate from pigs carrying Salmonella into the slaughterhouse. The observation that some carcasses at the re-work station contained MLVA types that were not detected on the same carcass at exsanguination, again showed that cross contamination from one carcass to another can also have occurred.

In this study the carcass splitter was identified as an important source of $S$. Rissen contamination. In previous assessments the carcass splitter has been considered an unimportant attributive source of Salmonella, because of the high infection status of the pigs entering the slaughterhouse, especially, if the splitter is equipped with automatic disinfection between each carcass and faecal contamination during evisceration is controlled (Berends et al., 1997; Borch et al., 1996). However, other reports showed that a significant Salmonella contamination via the slaughterhouse environment was caused by the carcass splitter 
(Sørensen et al., 1999; Swanenburg et al., 2001a, 2001b). In the present study slaughter equipment apparently contributed also to Salmonella on pig carcasses. Despite cleaning and disinfection, one of the robots was repeatedly contaminated with S. Rissen. Moreover, once this serovar was even present on this carcass splitter prior to the start of slaughter on that day and over the weekend (Fig 3; S. Rissen; CS, 13-07-2009).

In the slaughterhouse studied, cross-contamination contributed significantly to the carcass contamination. Resident flora was detected throughout the study on one of the slaughter robots. The serovar identified, $S$. Rissen, contributed significantly to the contamination at the end of the slaughter-line, whereas it was not found on any of the incoming carcasses. In addition, serovars on carcass at the re-work station were many times other types than the ones detected at exsanguination in skin and faeces samples. The data collected, especially the Salmonella enumeration results and the sero- as well as genotyping data, gave unique insight in the dynamics of transmission in a slaughter-line.

The sero- and genotyping data will be compared using a variety of statistical tests and implemented in a tracing scheme to predict the source of Salmonella on a carcass at the rework station (Smid et al., 2011). 


\section{Acknowledgements}

387 This work was supported by the European Union funded Integrated Research Project BIOTRACER (contract FOOD-2006-CT-036272) under the 6th RTD Framework.

We would like to thank Paul Hengeveld for his help during sampling at the slaughterhouse. 


\section{References}

Alban, L., Stärk, K.D.C., 2005. Where should the effort be put to reduce the Salmonella prevalence in the slaughtered swine carcass effectively? Preventive Veterinary Medicine $68,63-79$.

Arguello, H., Carvajal, A., Collazos, J.A., García-Feliz, C., Rubio, P., 2011. Prevalence and serovars of Salmonella enterica on pig carcasses, slaughtered pigs and the environment of four Spanish slaughterhouses. Food Research International, in press.

Baptista, F.M., Dahl, J., Nielsen, L.R., 2010. Factors influencing Salmonella carcass prevalence in Danish pig abattoirs. Preventive Veterinary Medicine 95, 231-238.

Berends, B.R., van Knapen, F., Snijders, J.M.A., Mossel, D.A.A., 1997. Identification and quantification of risk factors regarding Salmonella spp. on pork samples. International Journal of Food Microbiology 36, 199-206.

Borch, E., Nesbakken, T., Christensen, H., 1996. Hazard identification in swine slaughter respect to foodborne bacteria. International Journal of Food Microbiology 30, 9-25.

Botteldoorn, N., Heyndrickx, M., Rijpens, N., Grijspeerdt, K., Herman, L., 2003. Salmonella on pig carcasses: positive pigs and cross contamination in the slaughterhouse. Journal of Applied Microbiology 95, 891-903.

Bouvet, J., Bavai, C., Rossel, R., Le Roux, A., Montet, M.P., Mazuy, C., Vernozy-Rozand, C., 2003. Evolution of pig carcass and slaughterhouse environment contamination by Salmonella. Revue de Médecine Vétérinaire 154, 775-779.

De Busser, E.V., Maes, D., Houf, K., Dewulf, J., Imberechts, H., Bertrand, S., De Zutter, L., 2011. Detection and characterization of Salmonella in lairage, on pig carcasses and intestines in five slaughterhouses. International Journal of Food Microbiology 145, 279_ 286. 
De Man, J.C., 1983. "MPN Tables, Corrected." European Journal of Applied Microbiology and Biotechnology 17, 301-305.

EFSA, European Food Safety Authority, 2006. Opinion of the scientific panel on biological hazards on "Risk assessment and mitigation options of Salmonella in pig production". The EFSA Journal 341, 1-131.

EFSA, European Food Safety Authority, 2008. Report of the task force on zoonoses data collection on the analysis of the baseline survey on the prevalence of Salmonella in slaughter pigs, Part A. The EFSA Journal 135, 1-111.

EFSA, European Food Safety Authority, 2010. The community summary report on trends and sources of zoonoses, zoonotic agents and food-borne outbreaks in the European Union in 2008. The EFSA Journal 8, 1496.

Gelman, A., Carlin, J.B., Stern, H.S., Rubin, D.B., 2004. Bayesian data analysis, Chapman and Hall/CRC, London, UK.

Grimont, P.A.D., Weill F.-X., 2007. Antigenic Formulae of the Salmonella Serovars (9th ed.). WHO Collaborating Center for Reference and Research on Salmonella, Institut Pasteur, Paris.

Hald, T., Wingstrand, A., Swanenburg, M., von Altrock, A., Thorberg, B.-M., 2003. The occurrence and epidemiology of Salmonella in European pig slaughterhouses. Epidemiology and Infection 131, 1187-1203.

Hutchison, M.L., Walters, L.D., Avery, S.M., Reid, C.-A., Wilson, D., Howell, M., Johnston, A.M., Buncic, S., 2005. A comparison of wet-dry swabbing and excision sampling methods for microbiological testing of bovine, porcine, and ovine carcasses at red meat slaughterhouses. Journal of Food Protection 68, 2155-2162.

Lim, Y.-H., Hirose, K., Izumiya, H., Arakawa, E., Takahashi, H., Terajima, J., Itoh, K., Tamura, K., Kim S.-I., Watanabe, H., 2003. Multiplex polymerase chain reaction assay for 
selective detection of Salmonella enterica serovar Typhimurium. Japanese Journal of Infectious Diseases 56, 151-155.

Lindblad, M., 2007. Microbiological sampling of swine carcasses: A comparison of data obtained by swabbing with medical gauze and data collected routinely by excision at Swedish abattoirs. International Journal of Food Microbiology 118, 180-185.

Lindstedt, B.-A., Vardund, T., Aas, L., Kapperud, G., 2004. Multiple-locus variable-number tandem-repeats analysis of Salmonella enterica subsp. enterica serovar Typhimurium using PCR multiplexing and multicolor capillary electrophoresis. Journal of Microbiological Methods 59, 163-172.

Malorny, B., Paccassoni, E., Fach, P., Bunge, C., Martin, A., Helmuth, R., 2004. Diagnostic real-time PCR for detection of Salmonella in food. Applied and Environmental Microbiology 70, 7046-7052.

Martínez, B., Celda, M.F., Anastasio, B., García, I., López-Mendoza, M.C., 2010. Microbiological sampling of carcasses by excision or swabbing with three types of sponge or gauze. Journal of Food Protection 73, 81-87.

Oosterom, J., Dekker, R., de Wilde, G.J., van Kempen-de Troye, F., Engels, G.B., 1985. Prevalence of Campylobacter jejuni and Salmonella during pig slaughtering. Veterinary Quarterly 7, 31-34.

Ribot, E.M., Fair, M.A., Gautom, R., Cameron, D.N., Hunter, S.B., Swaminathan, B., Barrett, T.J., 2006. Standardization of pulsed-field gel electrophoresis protocols for the subtyping of Escherichia coli O157:H7, Salmonella, and Shigella for PulseNet. Foodborne Pathogens and Disease 3, 59-67.

Smid, J.H., van Hoek, A. H. A. M., Aarts, H.J.M., Havelaar, A.H., Heres, L., de Jonge, R., Pielaat A., 2011. Quantifying the sources of Salmonella contamination in a Dutch pig slaughter plant. Journal of Applied Microbiology, submitted. 
Sørensen, L.L., Sørensen, R., Klint, K., Nielsen, B., 1999. Persistent environmental strains of Salmonella infantis at two Danish slaughterhouses, two case-stories. In: Proceedings of the 3rd International Symposium on Epidemiology and Control of Salmonella in Pork, Washington, DC, 4-7 August, pp. 285-286.

Swanenburg, M., Urlings, H.A.P., Snijders, J.M.A., Keuzenkamp, D.A. van Knapen, F., 2001a. Salmonella in slaughter pigs: prevalence, serotypes and critical control points during slaughter in two slaughterhouses International Journal of Food Microbiology 70, 243-254.

Swanenburg, M., van der Wolf, P.J., Urlings, H.A.P., Snijders J.M.A., van Knapen, F., 2001b. Salmonella in slaughter pigs: the effect of logistic slaughter procedures of pigs on the prevalence of Salmonella in pork. International Journal of Food Microbiology 70, 231242.

Torpdahl, M., Sorensen, G., Lindstedt, B.A., Nielsen, E.M., 2007. Tandem repeat analysis for surveillance of human Salmonella Typhimurium infections. Emerging Infectious Diseases 13, 388-395.

Valdezate, S., Vidal, A., Herrera-Leon, S., Pozo, J., Rubio, P., Usera, M.A., Carvajal, A., Echeita, M.A., 2005. Salmonella Derby clonal spread from pork. Emerging Infectious Diseases 11, 694-698.

Valkenburgh, S., van Oosterom, R., Stenvers, O., Aalten, M., Braks, M., Schimmer, B., van de Giessen, A., van Pelt, W., Langelaar, M. 2007. Zoonoses and zoonotic agents in humans, food, animals and feed in The Netherlands 2003-2006. (http://www.rivm.nl/bibliotheek/rapporten/330152001.pdf)

van Pelt, W., van Giessen, A., van Leeuwen, W., Wannet, W., Henken, A., Evers, E., de Wit, M., van Duynhoven, Y., 2000. Oorsprong, omvang en kosten van humane salmonellose. Infectieziekten Bulletin 11, 4-8. 
489 Visscher, C.F., Klein, G., Verspohl, J., Beyerbach, M., Stratmann-Selke, J., Kamphues, J., 490 2011. Serodiversity and serological as well as cultural distribution of Salmonella on farms and in abattoirs in Lower Saxony, Germany. International Journal of Food Microbiology $146,44-51$.

Vose, D., 2000. Risk analysis, A quantitative guide. John Wiley \& Sons Ltd, Chichester, $494 \quad$ United Kingdom.

495 Warriner, K., Aldsworth, T.G., Kaur, S., Dodd, C.E.R., 2002. Cross-contamination of carcasses and equipment during pork processing. Journal of Applied Microbiology 93, $169-177$. 
Figure Legends

499

Fig. 1.

500

Locations of the various cork borer samples, rectal and equipment swabs taken during the slaughtering process in the pig slaughterhouse investigated.

Fig. 2.

504 Salmonella prevalence data at the different carcass sampling sites determined by real-time PCR. The black bars represent the samples taken at approximately $11 \mathrm{AM}$; the grey ones indicate the samples taken at approximately 1PM; the white bars show the samples taken at approximately 3PM.

Fig. 3.

510 PFGE dendrogram of $S$. Rissen and $S$. Derby isolates from the slaughter-line and pigs. BO: Belly opener; CS: Carcass splitter; EE: Exsanguination, exterior; FS: Rectal swab; RE: Re-

512 work station, exterior; RI: Re-work station, interior.

513 Sample names in bold indicate $S$. Derby isolates from individual carcasses isolated at different 514 stages of the slaughter-line. 
Figure 1.

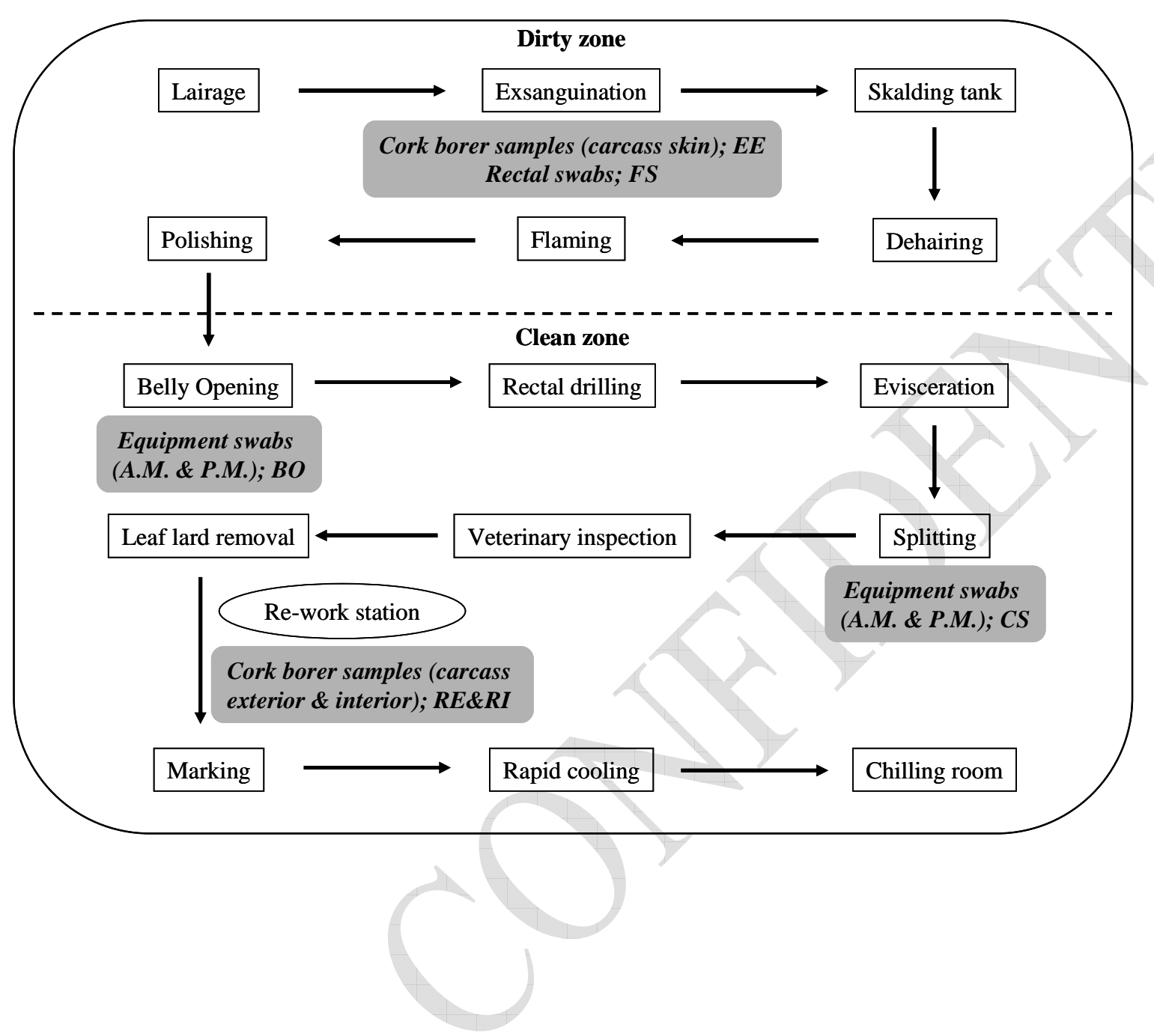


Figure 2.

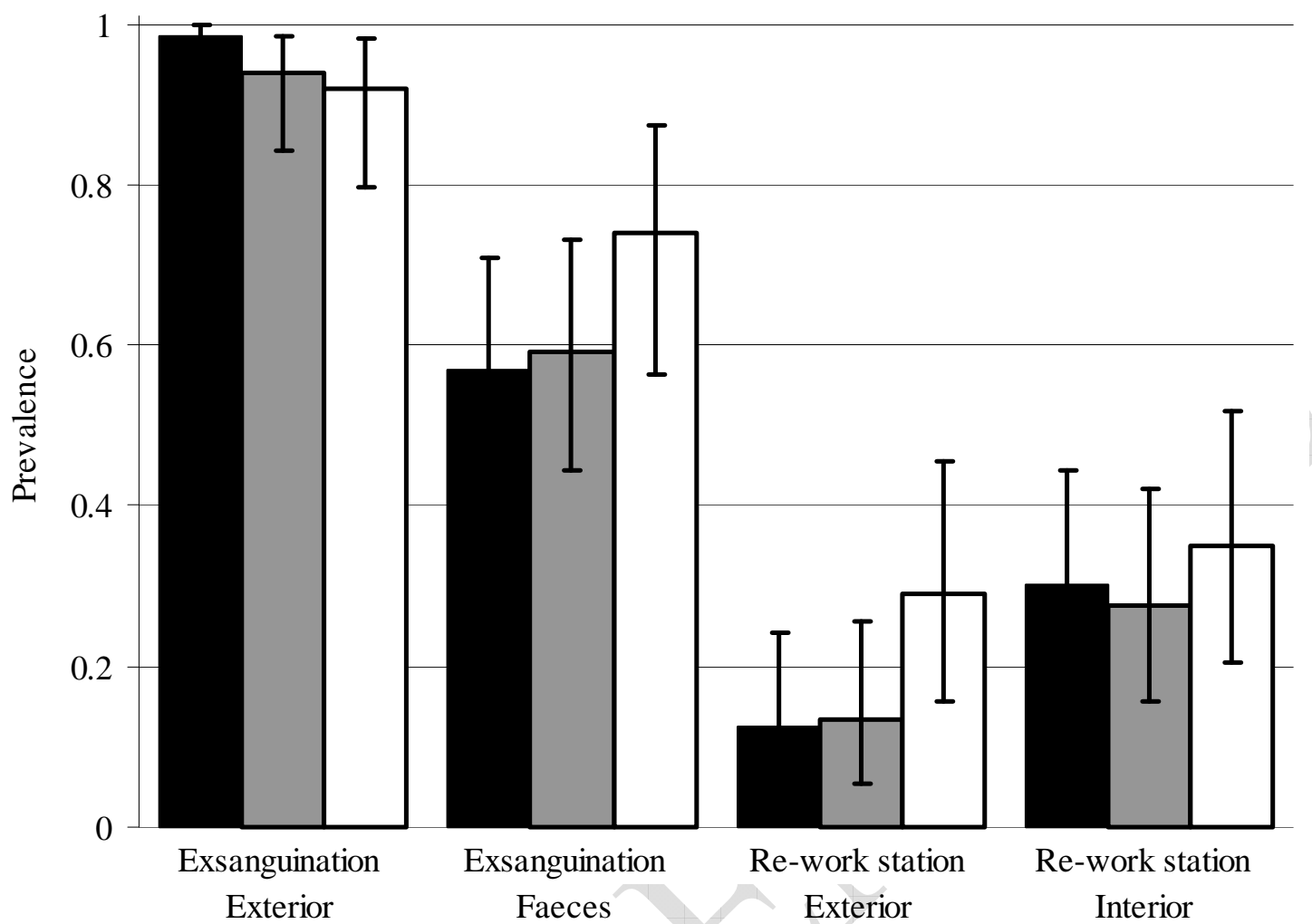




\section{$517 \quad$ Figure 3.}

S. Rissen_RE_08-06-2009 (Animal 199_1) S. Rissen_RI_08-06-2009 (Animal 119_1) S. Rissen_RE_08-06-2009 (Animal 195_1) $S$. Rissen_RI 08-06-2009 (Animal 195_1) $S$. Rissen RE 15-06-2009 (Animal 723 1) $S$. Rissen_RI_15-06-2009 (Animal 723_1) $S$. Rissen_RE_15-06-2009 (Animal 011_1) S. Rissen_RI_15-06-2009 (Animal 396_2) $S$. Rissen_RI_15-06-2009 (Animal 518_1) $S$. Rissen_RI_16-06-2009 (Animal 662_1) S. Rissen_RI_13-07-2009 (Animal 867_1)

$S$. Rissen_CS_02-06-2009

$S$. Rissen_CS_02-06-2009

$S$. Rissen_CS_08-06-2009

S. Rissen_CS_08-06-2009

$S$. Rissen CS 09-06-2009

$S$. Rissen_CS_15-06-2009

$S$. Rissen_CS_15-06-2009

S. Rissen_CS_15-06-2009

S. Rissen_CS_16-06-2009

S. Rissen CS 13-07-2009

S. Rissen_CS_13-07-2009

$S$. Rissen_CS_13-07-2009

S. Rissen_CS_09-06-2009

S. Rissen_CS_14-07-2009

$S$. Derby_CS 08-06-2009

S. Derby CS 15-06-2009

$S$. Derby_CS_16-06-2009

$S$. Derby_CS_16-06-2009

$S$. Derby_CS_13-07-2009

$S$. Derby_CS_15-06-2009

S. Derby_RI_02-06-2009 (Animal 942_1)

$S$. Derby_CS_16-06-2009

$S$. Derby_EE_02-06-2009 (Animal 171_1)

$S$. Derby_EE_02-06-2009 (Animal 826_1)

$S$. Derby_FS_16-06-2009 (Animal 687_2)

S. Derby_FS 16-06-2009 (Animal 657 2)

$S$. Derby_EE 15-06-2009 (Animal 723 2)

$S$. Derby_EE_15-06-2009 (Animal 011_1)

$S$. Derby_EE_15-06-2009 (Animal 518_1)

S. Derby_EE_15-06-2009 (Animal 787_1)

$S$. Derby FS 15-06-2009 (Animal 787 1)

$S$. Derby EE 15-06-2009 (Animal 787_2)

S. Derby_EE_25-05-2009 (Animal 011_1)

$S$. Derby_EE_25-05-2009 (Animal 723_2)

$S$. Derby_EE_02-06-2009 (Animal 292_2)

$S$. Derby_RI 02-06-2009 (Animal 171 1)

$S$. Derby_EE 02-06-2009 (Animal 171_2)

S. D.

S. Derby_EE_02-06-2009 (Animal 942_1)

(Animal 942_1)

$S$. Derby_EE_02-06-2009 (Animal 592_1)

$S$. Derby_EE_02-06-2009 (Animal 592_2)

$S$. Derby_EE 02-06-2009 (Animal 875_1)

$S$. Derby_EE 02-06-2009 (Anima1 723_1)

S. D.

$S$. Derby_RI_08-06-2009 (Animal 921_1)
$S$. Derby_RI_08-06-2009 (Animal 968_1)

S. Derby_RI_08-06-2009 (Animal 968_1)

$S$. Derby_RE_08-06-2009 (Animal 119_1)

$S$. Derby_RE 08-06-2009 (Animal 611_1)

S. Derby RI 08-06-

S. Derby_RI_08-06-2009 (Animal 195_1)

S. Derby_RI_08-06-2009 (Animal 195_1)

S. Derby_RI_08-06-2009 (Animal 723_2)

S. Derby_RI 09-06-2009 (Animal 325 2)

$S$. Derby RI 15-06-2009 (Animal 921_1)

S. Derby_EE 15-06-2009 (Animal 723 1)

S. Derby_RI_15-06-2009 (Animal 532_1)

$S$. Derby_RI_15-06-2009 (Animal 787_2)

S. Derby_RI_15-06-2009 (Animal 921_2)

$S$. Derby CS 25-05-2009

$S$. Derby_CS 25-05-2009

$S$. Derby_CS_02-06-2009

$S$. Derby_CS_02-06-2009

$S$. Derby_CS 09-06-2009

$S$. Derby_CS_09-06-2009

S. Derby_CS_15-06-2009

S. Derby_CS_15-06-2009

S. Derby_CS_13-07-2009

S. Derby_CS_14-07-2009

S. Derby_RI_15-06-2009 (Animal 011_1)

$S$. Derby BO 16-06-2009

$S$. Derby CS 15-06-2009

$S$. Derby_CS_14-07-2009

S. Derby_FS_13-07-2009 (Animal 787_1)

$S$. Derby_CS 08-06-2009

$S$. Derby_RI_16-06-2009 (Animal 244_1)

S. Derby_EE_13-07-2009 (Animal 386_2)

$S$. Derby_EE_16-06-2009 (Animal 244_1)

$S$. Derby_RE_16-06-2009 (Animal 657_2

$S$. Derby_FS_16-06-2009 (Animal 657_2)

$S$. Derby_EE 14-07-2009 (Animal 588 1)

$S$. Derby EE 14-07-2009 (Anima1 588_2)

S. Derof

S. Derby_FS_14-07-2009 (Animal 588_2)

$S$. Derby_EE_16-06-2009 (Animal 657_1)

$S$. Derby_EE_08-06-2009 (Animal 968_1)

$S$. Derby_EE_08-06-2009 (Animal 119_1) 
Table 1: Number of Salmonella positive samples per sampling date and per sampling site determined by real-time PCR.

\begin{tabular}{|c|c|c|c|c|c|c|c|c|c|c|c|c|}
\hline \multirow[b]{2}{*}{ Date } & \multicolumn{4}{|c|}{ Robots - Before slaughter ${ }^{a}$} & \multicolumn{2}{|c|}{ Exsanguination } & \multicolumn{2}{|c|}{ Re-work station } & \multicolumn{4}{|c|}{ Robots - After slaughter ${ }^{a}$} \\
\hline & BO1 & $\mathrm{BO} 2$ & CS1 & $\mathrm{CS} 2$ & $\begin{array}{l}\text { Carcass } \\
\text { skin }\end{array}$ & Faeces & Exterior & Interior & BO1 & $\mathrm{BO} 2$ & CS1 & $\mathrm{CS} 2$ \\
\hline 14-04-2009 & nd & nd & nd & nd & $6 / 6$ & nd & $2 / 6$ & $1 / 6$ & $0 / 1$ & $0 / 1$ & $0 / 1$ & $0 / 1$ \\
\hline 20-04-2009 & $0 / 1$ & $0 / 1$ & $0 / 1$ & $0 / 1$ & $12 / 12$ & $10 / 12$ & $0 / 12$ & $0 / 12$ & $0 / 1$ & $0 / 1$ & $0 / 1$ & $0 / 1$ \\
\hline $11-05-2009$ & $0 / 1$ & $0 / 1$ & $0 / 1$ & $0 / 1$ & $8 / 8$ & $5 / 8$ & $0 / 8$ & $0 / 8$ & $0 / 2$ & $0 / 2$ & $0 / 2$ & $0 / 2$ \\
\hline 25-05-2009 & $0 / 1$ & $0 / 1$ & $0 / 1$ & $0 / 1$ & $11 / 12$ & $5 / 12$ & $2 / 11$ & $0 / 11$ & $0 / 2$ & $0 / 2$ & $0 / 2$ & $2 / 2$ \\
\hline 02-06-2009 & $0 / 1$ & $0 / 1$ & $0 / 1$ & $0 / 1$ & $12 / 12$ & $6 / 12$ & $0 / 12$ & $3 / 12$ & $0 / 2$ & $0 / 2$ & $0 / 2$ & $2 / 2$ \\
\hline 08-06-2009 & $0 / 1$ & $0 / 1$ & $0 / 1$ & $0 / 1$ & $12 / 12$ & $11 / 12$ & $5 / 12$ & $8 / 12$ & $0 / 2$ & $0 / 2$ & $0 / 3$ & $3 / 3$ \\
\hline 09-06-2009 & $0 / 1$ & $0 / 1$ & $0 / 1$ & $0 / 1$ & $8 / 8$ & $6 / 8$ & $2 / 8$ & $3 / 8$ & $0 / 2$ & $1 / 2$ & $0 / 2$ & $2 / 2$ \\
\hline 15-06-2009 & $0 / 1$ & $0 / 1$ & $0 / 1$ & $0 / 1$ & $12 / 12$ & $8 / 12$ & $2 / 12$ & $8 / 12$ & $0 / 2$ & $0 / 2$ & $0 / 4$ & $4 / 4$ \\
\hline 16-06-2009 & nd & nd & nd & nd & $12 / 12$ & $8 / 12$ & $2 / 12$ & $7 / 12$ & $1 / 2$ & $1 / 2$ & $0 / 3$ & $3 / 3$ \\
\hline 13-07-2009 & $0 / 2$ & $0 / 2$ & $0 / 2$ & $2 / 2$ & $12 / 12$ & $8 / 12$ & $3 / 12$ & $2 / 12$ & $0 / 2$ & $0 / 2$ & $0 / 2$ & $2 / 2$ \\
\hline 14-07-2009 & $0 / 2$ & $0 / 2$ & $0 / 2$ & $2 / 2$ & $9 / 12$ & $3 / 12$ & $1 / 12$ & $3 / 12$ & $0 / 2$ & $0 / 2$ & $0 / 2$ & $2 / 2$ \\
\hline Total & $0 / 11$ & $0 / 11$ & $0 / 11$ & $4 / 11$ & $114 / 118$ & $70 / 112$ & $19 / 117$ & $35 / 117$ & $1 / 20$ & $2 / 20$ & $0 / 24$ & $20 / 24$ \\
\hline
\end{tabular}


Table 2. Estimated parameters (sample mean, $\hat{\mu}$, and standard error, $\hat{\sigma}$ ) of the Log-Normal

521 probability distribution representing the concentration of positive samples.

\begin{tabular}{|c|c|c|c|c|c|c|c|c|}
\hline \multirow[b]{3}{*}{ Date } & \multicolumn{4}{|c|}{ Exsanguination } & \multicolumn{4}{|c|}{ Re-work station } \\
\hline & \multicolumn{2}{|c|}{$\begin{array}{l}\text { Carcass skin (log } \\
\left.\text { MPN/cm }{ }^{2}\right)\end{array}$} & \multicolumn{2}{|c|}{$\begin{array}{l}\text { Faeces (log } \\
\text { MPN/g) }\end{array}$} & \multicolumn{2}{|c|}{$\begin{array}{l}\text { Exterior (log } \\
\left.\text { MPN/ } \mathrm{cm}^{2}\right)\end{array}$} & \multicolumn{2}{|c|}{$\begin{array}{l}\text { Interior }(\log \\
\text { MPN/cm²) }\end{array}$} \\
\hline & $\hat{\mu}$ & $\hat{\sigma}$ & $\hat{\mu}$ & $\hat{\sigma}$ & $\hat{\mu}$ & $\hat{\sigma}$ & $\hat{\mu}$ & $\hat{\sigma}$ \\
\hline 14-04-2009 & 1.75 & 0.59 & $\overline{\text { nd }}$ & & $<-0.51$ & & $<-0.35$ & \\
\hline 20-04-2009 & 0.47 & 0.49 & 2.71 & 0.98 & & & & \\
\hline $11-05-2009$ & 0.42 & 0.71 & 1.91 & 1.19 & & & & \\
\hline $25-05-2009$ & 0.26 & 0.75 & 2.31 & 1.36 & $<-0.79$ & & & \\
\hline 02-06-2009 & 0.46 & 0.84 & 2.7 & 0.68 & & & $<-0.61$ & \\
\hline 08-06-2009 & 0.04 & 0.91 & 2.11 & 1.02 & 0.11 & 0.53 & -0.13 & 1.12 \\
\hline 09-06-2009 & 0.52 & 0.71 & 2.35 & 0.78 & $<-0.52$ & ( & -0.47 & 1.04 \\
\hline $15-06-2009$ & 0.60 & 0.61 & -3.32 & 4.96 & -0.42 & 0.82 & -0.31 & 0.72 \\
\hline $16-06-2009$ & 0.92 & 1.33 & 2.75 & 1.43 & $<-0.80$ & & -0.32 & 0.43 \\
\hline 13-07-2009 & 0.59 & 1.00 & 2.61 & 1.22 & -0.98 & 0.47 & -0.37 & 0.99 \\
\hline 14-07-2009 & 0.34 & 0.34 & 2.65 & 0.6 & $<-0.83$ & & $<-0.5 c$ & \\
\hline Average & 0.58 & 0.75 & 1.88 & 1.42 & -0.43 & 0.61 & -0.32 & 0.86 \\
\hline
\end{tabular}

Per month:

\begin{tabular}{|c|c|c|c|c|c|c|}
\hline \multirow[b]{2}{*}{ Date } & $\begin{array}{l}\text { Exsanguination } \\
\text { Carcass skin (log } \\
\text { MPN/cm²) }\end{array}$ & \multirow{2}{*}{$\begin{array}{l}\text { Faeces (log } \\
\text { MPN/g) }_{\hat{\mu}} \hat{\sigma}\end{array}$} & \multicolumn{2}{|c|}{$\begin{array}{l}\text { Re-work station } \\
\text { Exterior }(\log \\
\text { MPN/cm²) }\end{array}$} & \multicolumn{2}{|c|}{$\begin{array}{l}\text { Interior }(\log \\
\left.\text { MPN/cm } / \mathrm{cm}^{2}\right)\end{array}$} \\
\hline & $\hat{\mu} \quad \hat{\sigma}$ & & $\hat{\mu}$ & $\hat{\sigma}$ & $\hat{\mu}$ & $\hat{\sigma}$ \\
\hline April-May & 0.80 & $\begin{array}{ll}2.43 & 1.08\end{array}$ & $<-0.51$ & & $<-0.35$ & \\
\hline May-June & 0.31 & 2.33 & -0.38 & 0.69 & -0.49 & 1.09 \\
\hline June-July & 0.63 & 1.54 & -1.30 & 1.03 & -0.39 & 0.64 \\
\hline
\end{tabular}


Table 3. Salmonella serovars per sampling day determined by multiplex PCR and serotyping.

\begin{tabular}{lllllllll}
\hline & Serovar $^{\text {a }}$ & & & & & & \\
Date & BDY & BEG & DRB & INS & mSTM & RSN & STM & Unknown \\
\hline $14-04-2009$ & 0 & 0 & 4 & 6 & 14 & 0 & 3 & 0 \\
$20-04-2009$ & 0 & 0 & 55 & 1 & 2 & 0 & 1 & 0 \\
$11-05-2009$ & 0 & 0 & 20 & 0 & 6 & 0 & 8 & 0 \\
$25-05-2009$ & 0 & 0 & 4 & 0 & 0 & 0 & 16 & 0 \\
$02-06-2009$ & 0 & 0 & 35 & 0 & 1 & 5 & 11 & 0 \\
$08-06-2009$ & 5 & 16 & 33 & 0 & 0 & 13 & 26 & 1 \\
$09-06-2009$ & 0 & 32 & 12 & 0 & 0 & 4 & 14 & 0 \\
$15-06-2009$ & 1 & 6 & 41 & 0 & 0 & 20 & 12 & 0 \\
$16-06-2009$ & 0 & 0 & 27 & 0 & 0 & 6 & 42 & 1 \\
$13-07-2009$ & 3 & 30 & 5 & 0 & 0 & 13 & 24 & 0 \\
$14-07-2009$ & 0 & 0 & 19 & 0 & 0 & 2 & 22 & 0 \\
\% of total & $\mathbf{1 . 4 \%}$ & $\mathbf{1 3 . 5 \%}$ & $\mathbf{4 1 . 0 \%}$ & $\mathbf{1 . 1 \%}$ & $\mathbf{3 . 7 \%}$ & $\mathbf{1 0 . 1 \%}$ & $\mathbf{2 8 . 8 \%}$ & $\mathbf{0 . 3 \%}$ \\
\hline
\end{tabular}

Note: The multiplex PCR has been described by Lim et al. (2003). The serotyping was

526 performed by slide and tube agglutination following the Kauffmann-White scheme. ${ }^{a}$ BDY: $S$.

527 Bredeney; BEG: $S$. Brandenburg; DRB: S. Derby; INS: S. Infantis;

528 mSTM: monophasic variant $S$. Typhimurium; RSN: $S$. Rissen; STM: $S$. Typhimurium. 
Table 4. Salmonella serovars per sampling site determined by multiplex PCR and serotyping.

\begin{tabular}{|c|c|c|c|c|c|c|c|c|c|}
\hline \multirow{2}{*}{ Sample place ${ }^{\mathrm{b}}$} & & \multicolumn{8}{|c|}{ Serotype $^{\mathrm{a}}$} \\
\hline & & BDY & BEG & DRB & INS & mSTM & RSN & STM & Unknown \\
\hline \multirow{4}{*}{$\begin{array}{l}\text { Robots - } \\
\text { Before slaughter }\end{array}$} & BO1 & 0 & 0 & 0 & 0 & 0 & 0 & 0 & 0 \\
\hline & $\mathrm{BO} 2$ & 0 & 0 & 0 & 0 & 0 & 0 & 0 & 0 \\
\hline & SP1 & 0 & 0 & 0 & 0 & 0 & 0 & 0 & 0 \\
\hline & SP2 & 0 & 0 & 2 & 0 & 0 & 6 & 0 & 0 \\
\hline \multirow[t]{2}{*}{ Exsanguination } & Skin & 7 & 63 & 118 & 6 & 17 & 0 & 97 & 1 \\
\hline & Faeces & 2 & 17 & 47 & 1 & 2 & 0 & 60 & 0 \\
\hline \multirow[t]{2}{*}{ Re-work station } & Carcass exterior & 0 & 3 & 5 & 0 & 3 & 10 & 8 & 0 \\
\hline & Carcass interior & 0 & 1 & 37 & 0 & 1 & 12 & 8 & 1 \\
\hline \multirow{4}{*}{$\begin{array}{l}\text { Robots - } \\
\text { After slaughter }\end{array}$} & BO1 & 0 & 0 & 1 & 0 & 0 & 0 & 0 & 0 \\
\hline & $\mathrm{BO} 2$ & 0 & 0 & 0 & 0 & 0 & 0 & 6 & 0 \\
\hline & $\mathrm{CS} 1$ & 0 & 0 & 0 & 0 & 0 & 0 & 0 & 0 \\
\hline & $\mathrm{CS} 2$ & 0 & 0 & 45 & 0 & 0 & 35 & 0 & 0 \\
\hline
\end{tabular}

Note: The multiplex PCR has been described by Lim et al. (2003). The serotyping was

531 performed by slide and tube agglutination following the Kauffmann-White scheme. ${ }^{a}$ BDY: $S$.

532 Bredeney; BEG: $S$. Brandenburg; DRB: $S$. Derby; INS: $S$. Infantis; mSTM: monophasic variant $S$.

533 Typhimurium; RSN: $S$. Rissen; STM: $S$. Typhimurium. ${ }^{\mathrm{b}} \mathrm{BO}=$ Belly opener, $\mathrm{CS}=$ Carcass splitter. 
534 Table 5: MLVA types distribution among the various samples.

\begin{tabular}{|c|c|c|c|c|c|c|c|}
\hline \multirow{2}{*}{ Serovar } & \multirow[b]{2}{*}{ Allele string } & \multirow[b]{2}{*}{ Date } & \multicolumn{2}{|c|}{ Exsanguination } & \multicolumn{3}{|c|}{ Re-work station } \\
\hline & & & Carcass skin & Faeces & Exterior & Interior & $\mathrm{BO} 2$ \\
\hline monophasic & $02-03-19-14-02$ & $02 / 06 / 2009$ & & 1 & & & \\
\hline \multirow[t]{3}{*}{ S. Typhimurium } & 02-06-04-00-02 & $14 / 04 / 2009$ & 4 & & 3 & 1 & \\
\hline & & 20/04/2009 & 1 & & & & \\
\hline & $02-07-06-00-02$ & $11 / 05 / 2009$ & 2 & 1 & & & \\
\hline \multirow[t]{32}{*}{ S. Typhimurium } & $02-02-05-00-02$ & 09/06/2009 & 3 & & 1 & & 1 \\
\hline & & 13/07/2009 & & & 1 & & \\
\hline & & $14 / 07 / 2009$ & 7 & & & 1 & \\
\hline & $02-03-19-01-02$ & 02/06/2009 & 1 & & & & \\
\hline & & 13/07/2009 & 1 & & & & \\
\hline & $02-03-19-14-02$ & $25 / 05 / 2009$ & 1 & & & & \\
\hline & & 02/06/2009 & 1 & 4 & & & \\
\hline & & 08/06/2009 & 5 & 5 & & & \\
\hline & & 09/06/2009 & & 1 & & & \\
\hline & & $15 / 06 / 2009$ & 4 & 2 & & & \\
\hline & & 13/07/2009 & 5 & 1 & 1 & & \\
\hline & $02-05-05-00-02$ & $11 / 05 / 2009$ & 5 & 2 & & & \\
\hline & $02-05-06-00-03$ & $25 / 05 / 2009$ & & 2 & & & \\
\hline & & 08/06/2009 & & 1 & & & \\
\hline & $02-05-20-00-02$ & $16 / 06 / 2009$ & 9 & 3 & & & \\
\hline & 02-06-04-00-02 & $14 / 04 / 2009$ & 1 & & & & \\
\hline & 02-07-09-08-03 & 16/06/2009 & 2 & 2 & 1 & 1 & 1 \\
\hline & $02-07-10-08-03$ & 16/06/2009 & . & 1 & & & \\
\hline & $02-07-11-06-03$ & 02/06/2009 & 1 & & & & \\
\hline & & 08/06/2009 & 1 & & & & \\
\hline & 02-08-09-05-03 & 20/04/2009 & & 1 & & & \\
\hline & $02-11-06-00-03$ & $14 / 07 / 2009$ & 1 & & & & \\
\hline & $02-17-05-00-02$ & $09 / 06 / 2009$ & 1 & & & & \\
\hline & $03-02-04-13-02$ & 08/06/2009 & & 2 & & & \\
\hline & $03-03-20-05-02$ & $14 / 07 / 2009$ & & & & 1 & \\
\hline & $03-04-04-22-02$ & $14 / 04 / 2009$ & 2 & & & & \\
\hline & & 02/06/2009 & & & & 1 & \\
\hline & & $16 / 06 / 2009$ & & & & 2 & \\
\hline & & $14 / 07 / 2009$ & & 1 & & & \\
\hline & $03-08-13-19-02$ & 14/07/2009 & & & 1 & & \\
\hline & $04-01-17-14-02$ & $25 / 05 / 2009$ & 8 & 3 & 2 & & \\
\hline & $06-03-00-00-01$ & 13/07/2009 & & 1 & & & \\
\hline
\end{tabular}


Table 6. All paired occurrences of (monophasic) $S$. Typhimurium typed by MLVA on single

carcasses.

\begin{tabular}{|c|c|c|c|c|}
\hline Date & Herd_Animal & Origin $^{\mathrm{a}}$ & Serovar $^{\mathrm{b}}$ & MLVA allele string \\
\hline \multirow[t]{2}{*}{ 14-04-2009 } & A_1 & EE-RE & mSTM & $02-06-04-00-02$ \\
\hline & $\mathrm{C}_{-} 1$ & EE-RE-RI & mSTM & 02-06-04-00-02 \\
\hline \multirow[t]{3}{*}{ 11-05-2009 } & 396_1 & EE-FS & mSTM & $02-07-06-00-02$ \\
\hline & 396_2 & EE-FS & STM & $02-05-05-00-02$ \\
\hline & $646 \_2$ & EE-FS & STM & $02-05-05-00-02$ \\
\hline \multirow[t]{5}{*}{ 25-05-2009 } & 723_1 & EE, FS & STM & $04-01-17-14-02,02-05-06-00-03$ \\
\hline & $787 \_1$ & EE-FS & STM & $04-01-17-14-02$ \\
\hline & 787_3 & EE-FS-RE & STM & $04-01-17-14-02$ \\
\hline & 900_1 & EE-FS & STM & $04-01-17-14-02$ \\
\hline & 900_2 & EE-RE & STM & $04-01-17-14-02$ \\
\hline 02-06-2009 & 826_1 & EE, FS & STM & 02-03-19-01-02, 02-03-19-14-02 \\
\hline \multirow[t]{5}{*}{ 08-06-2009 } & 431_1 & EE-FS & STM & $02-03-19-14-02$ \\
\hline & 611_2 & EE-FS & STM & 02-03-19-14-02 \\
\hline & 921_1 & EE, FS & STM & $02-03-19-14-02,03-02-04-13-02$ \\
\hline & $921 \_2$ & EE-FS & STM & $02-03-19-14-02$ \\
\hline & 968_1 & EE, FS & STM & 02-03-19-14-02, 03-02-04-13-02 \\
\hline \multirow[t]{2}{*}{$15-06-2009$} & $532 \_1$ & EE-FS & STM & $02-03-19-14-02$ \\
\hline & 921_1 & EE-FS & STM & $02-03-19-14-02$ \\
\hline \multirow[t]{6}{*}{$16-06-2009$} & $662 \_1$ & EE-FS & STM & $02-05-20-00-02$ \\
\hline & $662 \_2$ & EE-FS & STM & 02-05-20-00-02 \\
\hline & 657_1 & EE-FS, RI & STM & 02-05-20-00-02, 03-04-04-22-02 \\
\hline & 657_2 & EE, RI & STM & $02-05-20-00-02,03-04-04-22-02$ \\
\hline & $657 \_3$ & EE-FS & STM & 02-07-09-08-03 \\
\hline & $657 \_4$ & EE-FS-RE-1 & STM & 02-07-09-08-03 \\
\hline \multirow[t]{3}{*}{ 13-07-2009 } & $149 \_1$ & FS, RE & STM & 03-04-04-22-02, 03-08-13-19-02 \\
\hline & $921 \_1$ & EE-FS-RE & STM & $02-03-19-14-02$ \\
\hline & 921_2 & EE, FS & STM & $02-03-19-14-02,06-03-00-00-01$ \\
\hline
\end{tabular}

Note: ${ }^{a}$ EE: Exsanguination, exterior; FS: Exsanguination, Rectal swab; RE: Re-work station,

539 exterior; RI: Re-work station, interior. ${ }^{\text {b }}$ mSTM: monophasic $S$. Typhimurium; STM: $S$.

Typhimurium. 